\title{
Identification Via Quantum Channels
}

\author{
Andreas Winter \\ ICREA - Institució Catalana de Recerca i Estudis Avançats \\ Pg. Lluis Companys 23, ES-08010 Barcelona, Spain \\ Física Teòrica: Informació i Fenomens Quàntics \\ Universitat Autònoma de Barcelona, ES-08193 Bellaterra (Barcelona), Spain \\ Department of Mathematics, University of Bristol, Bristol BS8 1TW, U.K. \\ Centre for Quantum Technologies, National University of Singapore \\ 2 Science Drive 3, Singapore 117542, Singapore
}

\begin{abstract}
We review the development of the quantum version of Ahlswede and Dueck's theory of identification via channels. As is often the case in quantum probability, there is not just one but several quantizations: we know at least two different concepts of identification of classical information via quantum channels, and three different identification capacities for quantum information.

In the present summary overview we concentrate on conceptual points and open problems, referring the reader to the small set of original articles for details.
\end{abstract}

Dem Andenken an Rudolf Ahlwede (15/9/1938-18/12/2010)

\section{Quantum and classical channels}

Our communication model is the quantum channel, also known as completely positive and trace preserving (cptp) linear map between quantum systems,

$$
\mathcal{N}: \mathcal{L}(A) \longrightarrow \mathcal{L}(B) .
$$

Here, as in the rest of the paper, we assume that $A, B$, etc, are finite dimensional (complex) Hilbert spaces and $\mathcal{L}(A)$ is the set of linear operators (matrices) over A.

The cptp condition is necessary and sufficient for $\mathcal{N}$ mapping states on $A$, i.e. density operators $\rho \geq 0$ with $\operatorname{Tr} \rho=1$, whose set we denote as $\mathcal{S}(A)$, to states on $B$, and the same for $\mathcal{N} \otimes \operatorname{id}_{C}$ for arbitrary systems $C$. Thus, the class of cptp maps is closed under composition, tensor products and taking convex combinations. One of the most useful characterizations of cptp maps is in terms of the Stinespring dilation [29]: namely, $\mathcal{N}$ is cptp if and only if there exists 
an ancilla (environment) system $E$ and an isometry $V: A \hookrightarrow B \otimes E$ such that $\mathcal{N}(\rho)=\operatorname{Tr}_{E} V \rho V^{\dagger}$. The isometry $V$ is essentially unique, up to unitary equivalence of $E$; hence it makes sense to define, for a chosen dilation $V$, the complementary channel

$$
\widehat{\mathcal{N}}: \mathcal{L}(A) \longrightarrow \mathcal{L}(E)
$$

by $\widehat{\mathcal{N}}(\rho):=\operatorname{Tr}_{B} V \rho V^{\dagger}$.

For a given channel $\mathcal{N}$, we are interested in the asymptotic performance of many iid copies, $\mathcal{N}^{\otimes n}$. One can also consider more complicated channel models (such as with feedback, or with pre-shared correlations), but here we will restrict ourselves to the simple forward channel - see however 33. and [1].

Classical channels are of course transition probability kernels $N: \mathcal{X} \rightarrow \mathcal{Y}$ (with finite input and output alphabets $\mathcal{X}$ and $\mathcal{Y}$, respectively). Such a channel may be identified with the cptp map

$$
\begin{aligned}
\mathcal{N}: \mathcal{L}\left(\mathbb{C}^{\mathcal{X}}\right) & \longrightarrow \mathcal{L}\left(\mathbb{C}^{\mathcal{Y}}\right) \\
\rho & \longmapsto \sum_{x y} N(y \mid x)|y\rangle\langle x|\rho| x\rangle\langle y|,
\end{aligned}
$$

while a probability distribution $P$ on $\mathcal{X}$ is identified with the state $\sum_{x} P(x)|x\rangle\langle x|$.

Two special classes of channels we will have occasion to consider are the following, either whose input or whose output is classical: A cq-channel $\mathcal{N}$ : $\mathcal{X} \longrightarrow \mathcal{S}(B)$ is a cptp map of the form

$$
\mathcal{N}(\xi)=\sum_{x}\langle x|\xi| x\rangle \rho_{x},
$$

with states $\rho_{x}$ on $B$. A qc-channel $\mathcal{M}: \mathcal{S}(A) \longrightarrow \mathcal{Y}$ instead is given by a quantum measurement, i.e. a positive operator values measure (POVM) $\left(M_{y}\right)_{y \in \mathcal{Y}}$ such that $M_{y} \geq 0$ and $\sum_{y} M_{y}=1$. The channel then has the form

$$
\mathcal{M}(\rho)=\sum_{y} \operatorname{Tr} \rho M_{y}|y\rangle\langle y| .
$$

We refer the reader to the excellent text 31] for more details on quantum and classical channels, and the various transmission capacities associated with them, including their history. Here we need only two, the classical and the quantum capacity of a channel, $C(\mathcal{N})$ and $Q(\mathcal{N})$, respectively, defined as the maximum rates of asymptotically faithful transmission of classical bits and qubits, respectively, over many iid copies of the channel. They can be expressed as regularizations of entropic information quantities, based on the von Neumann entropy $S(\rho)=-\operatorname{Tr} \rho \log \rho$ of a quantum state $\rho$. They are given by the formulas

$$
\begin{aligned}
C(\mathcal{N}) & =\lim _{n \rightarrow \infty} \frac{1}{n} C^{(1)}\left(\mathcal{N}^{\otimes n}\right), \text { with } \\
C^{(1)}(\mathcal{N}) & =\max _{\left\{p_{x}, \rho_{x}\right\}} S\left(\sum_{x} p_{x} \mathcal{N}\left(\rho_{x}\right)\right)-\sum_{x} p_{x} S\left(\mathcal{N}\left(\rho_{x}\right)\right),
\end{aligned}
$$


and

$$
\begin{aligned}
Q(\mathcal{N}) & =\lim _{n \rightarrow \infty} \frac{1}{n} Q^{(1)}\left(\mathcal{N}^{\otimes n}\right), \text { with } \\
Q^{(1)}(\mathcal{N}) & =\max _{\rho \in \mathcal{S}(A)} S(\mathcal{N}(\rho))-S(\widehat{\mathcal{N}}(\rho)),
\end{aligned}
$$

both of which represent the culmination of concerted efforts of several researchers in the 1990s and early 2000s (Holevo-Schumacher-Westmoreland and Schumacher \& Lloyd-Shor-Devetak, respectively). The classical capacity generalizes Shannon's channel capacity for classical channels $N$, for which $C(N)=C^{(1)}(N)$ reduces to the famous formula in terms of the mutual information 28 .

The structure of the rest of the paper is as follows: In section 1 we present the definitions for identification of classical information via quantum channels, after Löber [25, generalizing the model of Ahlswede and Dueck [8]9. In section 2 we move to identification of quantum information; section 3 presents the recently developed theoretical underpinning to prove capacity formulas for two of the three quantum models. In section 1 we show how the quantum identification results imply new lower bounds on classical identification capacities, which we illustrate with several examples, shedding new light also on Löber's founding work [25]. Finally, section 5 is devoted to an outlook on open questions and possible conjectures.

\section{Classical Identification}

Ahlswede and Dueck 89.9 introduced identification by noting that while Shannon's theory of transmission presumes that the receiver wants to know everything about the message, in reality he may be interested only in certain aspects of it. In other words, the receiver may want to compute a function of the message. The most extreme case is that of identification: for sent message $m$ and an arbitrary message $m^{\prime}$, the receiver would like to be able to answer the question "Is $m=m^{\prime}$ ?" as accurately as possible.

Definition 1 (Löber [25]). A classical identification code for the channel $\mathcal{N}$ with error probability $\lambda_{1}$ of first, and $\lambda_{2}$ of second kind is a set $\left\{\left(\rho_{i}, D_{i}\right): i=\right.$ $1, \ldots, N\}$ of states $\rho_{i}$ on $A$ and operators $D_{i}$ on $B$ with $0 \leq D_{i} \leq 1$, i.e. the pair $\left(D_{i}, 11-D_{i}\right)$ forms a measurement, such that

$$
\begin{aligned}
\forall i & \operatorname{Tr}\left(\mathcal{N}\left(\rho_{i}\right) D_{i}\right) \geq 1-\lambda_{1}, \\
\forall i \neq j & \operatorname{Tr}\left(\mathcal{N}\left(\rho_{i}\right) D_{j}\right) \leq \lambda_{2} .
\end{aligned}
$$

For the special case of memoryless channels $\mathcal{N}^{\otimes n}$, we speak of an $\left(n, \lambda_{1}, \lambda_{2}\right)$-ID code, and denote the largest size $N$ of such a code $N\left(n, \lambda_{1}, \lambda_{2}\right)$.

An identification code as above is called simultaneous if all the $D_{i}$ are coexistent: this means that there exists a positive operator valued measure (POVM) $\left(E_{t}\right)_{t=1}^{T}$ and pairwise disjoint sets $\mathcal{D}_{i} \subset\{1, \ldots, T\}$ such that $D_{i}=\sum_{t \in \mathcal{D}_{i}} E_{t}$. The largest size of a simultaneous $\left(n, \lambda_{1}, \lambda_{2}\right)-I D$ code is denoted $N_{\operatorname{sim}}\left(n, \lambda_{1}, \lambda_{2}\right)$. 
Note that $N_{\operatorname{sim}}\left(n, \lambda_{1}, \lambda_{2}\right)=N\left(n, \lambda_{1}, \lambda_{2}\right)=\infty$ if $\lambda_{1}+\lambda_{2} \geq 1$, hence to avoid this triviality one has to assume $\lambda_{1}+\lambda_{2}<1$.

It is straightforward to verify that in the case of a classical channel, this definition reduces to the famous one of Ahlswede and Dueck 8], in particular all codes are without loss of generality automatically simultaneous. It was in fact Löber [25] in his $\mathrm{PhD}$ thesis who noticed that in the quantum case we have to make a choice - whether the receiver should be able to answer all or any one of the "Is the message $=m^{\prime}$ ?" questions. It was the original realization of Ahlswede and Dueck [8] that $N\left(n, \lambda_{1}, \lambda_{2}\right)$ grows doubly exponential in $n$, hence the following definition of the (classical) identification capacity:

Definition 2. The (simultaneous) classical ID-capacity of a quantum channel $\mathcal{N}$ is given by

$$
\begin{aligned}
C_{\mathrm{ID}}(\mathcal{N}) & =\inf _{\lambda>0} \liminf _{n \rightarrow \infty} \frac{1}{n} \log \log N(n, \lambda, \lambda), \\
C_{\mathrm{ID}}^{\operatorname{sim}}(\mathcal{N}) & =\inf _{\lambda>0} \liminf _{n \rightarrow \infty} \frac{1}{n} \log \log N_{\operatorname{sim}}(n, \lambda, \lambda),
\end{aligned}
$$

respectively. We say that the strong converse holds for the identification capacity if for all $\lambda_{1}+\lambda_{2}<1$,

$$
\lim _{n \rightarrow \infty} \frac{1}{n} \log \log N\left(n, \lambda_{1}, \lambda_{2}\right)=C_{\mathrm{ID}}(\mathcal{N}),
$$

and similarly for $C_{\mathrm{ID}}^{\mathrm{sim}}$.

Theorem 3 (Ahlswede/Dueck [8], Han/Verdu [18,19], Ahlswede [4]). For a classical channel $N$ and any $\lambda_{1}, \lambda_{2}>0$ with $\lambda_{1}+\lambda_{2}<1$,

$$
\lim _{n \rightarrow \infty} \frac{1}{n} \log \log N\left(n, \lambda_{1}, \lambda_{2}\right)=C(N),
$$

in particular, $C_{\mathrm{ID}}^{\operatorname{sim}}(N)=C_{\mathrm{ID}}(N)=C(N)$.

The direct part of the above theorem, due to Ahlswede and Dueck [8], can be seen by concatenating a sufficiently good Shannon channel code with an identification code for the ideal bit channel. For the latter, 8 contains a combinatorial construction showing that by $k$-bit encodings, one can identify $\geq 2^{\Omega\left(2^{k}\right)}$ messages. Using this, the direct part of the following result is immediate:

Theorem 4 (Löber [25], Ahlswede/Winter [10]). For quantum channel $\mathcal{N}$,

$$
C_{\mathrm{ID}}(\mathcal{N}) \geq C_{\mathrm{ID}}^{\mathrm{sim}}(\mathcal{N}) \geq C(\mathcal{N}) .
$$

The simultaneous ID-capacity obeys a strong converse under the additional restriction that the signal states $\rho_{i}$ are from a set that is the convex hull of $\leq 2^{2^{o(n)}}$ 
quantum states on $A^{n}$. I.e., denoting the maximum number of messages under this constraint by $\underline{N}_{\text {sim }}\left(n, \lambda_{1}, \lambda_{2}\right)$,

$$
\limsup _{n \rightarrow \infty} \frac{1}{n} \log \log \underline{N}_{\operatorname{sim}}\left(n, \lambda_{1}, \lambda_{2}\right) \leq C(\mathcal{N}),
$$

for $\lambda_{1}+\lambda_{2}<1$. (For instance, the $\rho_{i}$ could be restricted to be - approximately - separable states.)

For cq-channels, the constraint is w.l.o.g. satisfied since there are only $|\mathcal{X}|^{n}$ classical input symbols, so for these channels the simultaneous ID-capacity obeys a strong converse, with $C_{\mathrm{ID}}^{\operatorname{sim}}(\mathcal{N})=C(\mathcal{N})=C^{(1)}(\mathcal{N})$.

Indeed, in the case of cq-channels, the strong converse holds even without the simultaneity constraint:

$$
\lim _{n \rightarrow \infty} \frac{1}{n} \log \log N\left(n, \lambda_{1}, \lambda_{2}\right)=C(\mathcal{N})=C^{(1)}(\mathcal{N}),
$$

for $\lambda_{1}+\lambda_{2}<1$.

[To be precise, Löber's results are in the framework of Han and Verdú [18|19, of "arbitrary" sequences of channels and using information spectrum methods. As we are focusing on the iid case here, we stated only a special case of his theorem.]

The last, non-simultaneous part of the Theorem is the main identification result of [10, which was proved by developing a theory of tail bounds for sums of random matrices, extending classical Hoeffding bounds, and inspired by Ahlswede's strong converse for the ID-capacity of classical channels [4. The simplest, and most useful, version is as follows.

Lemma 5 (Ahlswede/Winter [10]). For i.i.d. random variables $X_{i}$ in $d \times d$ Hermitian matrices and $0 \leq X_{i} \leq \mathbb{1}$, such that $\mathbb{E} X_{i}=\mu 1$. Then, for $\mu \leq \alpha \leq 1$ and $0 \leq \alpha \leq \mu$, respectively,

$$
\begin{aligned}
& \operatorname{Pr}\left\{\frac{1}{n} \sum_{i=1}^{n} X_{i} \not \leq \alpha \mathbb{1}\right\} \leq d e^{-n D(\alpha \| \mu)}, \\
& \operatorname{Pr}\left\{\frac{1}{n} \sum_{i=1}^{n} X_{i} \geq \alpha \mathbb{1}\right\} \leq d e^{-n D(\alpha \| \mu)},
\end{aligned}
$$

with the binary relative entropy $D(\alpha \| \mu)=\alpha \ln \frac{\alpha}{\mu}+(1-\alpha) \ln \frac{1-\alpha}{1-\mu}$.

As a consequence, for all $0 \leq \epsilon \leq \frac{1}{2}$,

$$
\operatorname{Pr}\left\{\frac{1}{n} \sum_{i=1}^{n} X_{i} \notin[(1-\epsilon) \mu \mathbb{1},(1=\epsilon) \mu \mathbb{1}]\right\} \leq 2 d e^{-\frac{1}{4} n \mu \epsilon^{2}} .
$$

using elementary estimates for the relative entropy.

The power of this Lemma is in its giving explicit and simple tail bounds, useful already for finite $n$ and $d$, whereas general abstract large deviation theory - 
which applies, see [7] for a version in infinite dimension - often incurs complex finite $n$ behaviour, only yielding clear asymptotic statements. The proof of the Lemma is simple, too: it requires generalizing the elementary Markov-Chebyshev inequalities and the Bernstein trick from real random variables to matrices. It has since found countless applications in quantum information theory and beyond: The first proofs of some core results such as the quantum channel capacity, remote state preparation or decoupling heavily relied on it, cf. [31, as did the structurally simple proof of the Alon-Roichman theorem and matrix versions of compressed sensing, cf. [30] and references therein. The latter also presents far-reaching generalizations of the above bounds.

It is not known whether simultaneous and non-simultaneous ID-capacity coincide or not for general quantum channels. In any case, going beyond simultaneity seems to provide major freedom:

Example 6. Buhrman et al. [15] found that in the space of $n$ qubits, whilst the largest number of orthogonal pure state vectors is clearly the dimension of the Hilbert space, $2^{n}$, there are $N \geq 2^{\Omega\left(2^{n}\right)}$ pairwise almost orthogonal pure states, i.e. $\left|\left\langle\psi_{i} \mid \psi_{j}\right\rangle\right| \leq \epsilon$ for $i \neq j$.

They dubbed this "fingerprinting" because a verifier who gets a copy of each $\left|\psi_{i}\right\rangle$ and $\left|\psi_{j}\right\rangle$ can efficiently determine whether $i=j$ or not. In particular, the set of these vectors forms a (non-simultaneous) ID-code, with $\rho_{i}=D_{i}=\left|\psi_{i}\right\rangle\left\langle\psi_{i}\right|$.

One can obtain a set of such vectors also by turning the probability distributions on $n$ bits form [8] into superpositions - cf. 32 for details.

Fingerprinting ID-codes use quantum superpositions in a nontrivial way, albeit the almost-orthogonality is somewhat analogous to the way the classical distributions in 8 do not overlap too much. However, they only use pure states, whereas the power of classical identification comes from randomization. Hence it is natural to ask whether mixed states offer any improvement. As the classical capacity of a noiseless qubit channel is 1 , the following result came as a bit of a surprise. It was proved using powerful geometric measure concentration techniques - cf. 2111] for other applications in quantum information theory.

Theorem 7 (Winter [32]). For the noiseless qubit channel $\mathrm{id}_{2}=\operatorname{id}_{\mathbb{C}^{2}}$, and $0<\lambda_{1}, \lambda_{2}, \lambda_{1}+\lambda_{2}<1$,

$$
2^{\Omega\left(2^{2 n}\right)} \leq N\left(n, \lambda_{1}, \lambda_{2}\right) \leq 2^{O\left(2^{2 n}\right)} .
$$

As a consequence, $C_{\mathrm{ID}}\left(\mathrm{id}_{2}\right)=2$ and the strong converse holds. If the encodings are restricted to pure states, the capacity is only 1.

[In 32] (Remark 13; the technical argument there has been elaborated in [17]) it was heuristically argued that one would expect $C_{\mathrm{ID}}^{\operatorname{sim}}\left(\mathrm{id}_{2}\right)$ to be 1 rather than 2.]

To appreciate why this result was so surprising, we need to go back to the insights from the original identification papers 899: It was understood that what determines identification capacity of a communication system is its ability 
to establish common randomness (cf. [6]), as long as some sublinear amount of actual communication is available. But the common randomness capacity of a noiseless qubit channel is 1 . However, a noiseless qubit channel can also establish entanglement (ebits) at rate 1. And indeed, in 331 it was found that $k$ EPR pairs shared between sender and receiver, together with $o(n)$ bits of communication are sufficient to identify $2^{\Omega\left(2^{2 k}\right)}$ messages. In this respect, it may be interesting to draw attention to the following:

Proposition 8 (Winter [32]). Given an ID-code of rate $C$ and common randomness of rate $R$, one can construct an ID-code of rate $C+R-o(1)$ which uses the signal states of the first code and correlations with the common randomness.

In other words: Whatever your communication system, its identification capacity is increased by 1 by each bit of common randomness. This was used in 32 to derive a lower bound on the ID-capacity of a quantum channel: If $\mathcal{N}$ permits simultaneous transmission of classical bits and qubits at rates $C$ and $Q$, respectively, then $C_{\mathrm{ID}}(\mathcal{N}) \geq C+2 Q$. Thus the results of [16] become applicable, where the $Q-C$ capacity region was determined. As we saw above, this bound, can be strictly larger than the classical capacity $C(\mathcal{N})$ of the channel, marking a decisive departure from the behaviour of classical channels.

Beyond these bounds and a few special examples in 32, the ID-capacity (simultaneous or not) of a general quantum channel remains elusive. However, in section 4 below we shall present a new lower bound.

\section{How to Identify Quantum States?}

So far the only quantum element in the discussion pertained to the channel model. However, there is a natural way in which even the task of identification can be extended from classical to quantum information. This has been promoted in 32 and further in the more recent 22. In the following, $\mathcal{P}(A) \subset \mathcal{S}(A)$ denotes the set of pure quantum states on a system $A$.

Definition 9 (Winter [32]). A quantum ID-code for the channel $\mathcal{N}$ with error $\epsilon$, for the Hilbert space $K$, is a pair of maps $\mathcal{E}: \mathcal{P}(K) \longrightarrow \mathcal{S}(A)$ and $\mathcal{D}$ : $\mathcal{P}(K) \longrightarrow \mathcal{L}(B)$ with $0 \leq \mathcal{D}_{\varphi} \leq \mathbb{1}$ for all $\varphi=|\varphi\rangle\langle\varphi| \in \mathcal{P}(K)$, such that for all pure states/rank-one projectors $\psi, \varphi \in \mathcal{P}(K)$,

$$
\left|\operatorname{Tr} \psi \varphi-\operatorname{Tr} \mathcal{N}(\mathcal{E}(\psi)) \mathcal{D}_{\varphi}\right| \leq \epsilon
$$

If the encoding $\mathcal{E}$ is cptp we speak of a blind code, in general and to contrast it with the former, we call it visible.

For the case of an iid channel $\mathcal{N}^{\otimes n}$, we denote the maximum dimension of a blind (visible) quantum ID-code by $M(n, \epsilon)\left(M_{v}(n, \epsilon)\right)$.

This notion can be motivated as follows: In quantum transmission, the objective for the receiver is to recover the state $\psi$ by means of a suitable decoding 
(cptp) map $\widetilde{\mathcal{D}}: \mathcal{L}(B) \longrightarrow \mathcal{L}(K)$, with high accuracy. Of course then the receiver can perform any measurement on the decoded state, effectively simulating an arbitrary measurement on the original input state, in the sense that for any state $\rho$ and POVM $M=\left(M_{i}\right)_{i}$ on $K$, there exists another POVM $M^{\prime}=\left(M_{i}^{\prime}\right)_{i}$ on $B$ such that the measurement statistics of $\rho$ under $M$ is approximately that of $\mathcal{N}(\mathcal{E}(\rho))$ under $M^{\prime}$. $\left(M^{\prime}\right.$ can be written down directly via the adjoint $\widetilde{\mathcal{D}}^{\dagger}: \mathcal{L}(K) \longrightarrow \mathcal{L}(B)$ of the decoding map, which maps measurement POVMs on $K$ to POVMs on $B$ : $M_{i}^{\prime}=\widetilde{\mathcal{D}}^{\dagger}\left(M_{i}\right)$.) The converse is also true: If the receiver can simulate sufficiently general measurements on the input state by suitable measurements on the channel output, then he can actually decode the state by a cptp map $\widetilde{\mathcal{D}}[26$.

This allows us to relax the task of quantum information transmission to requiring only that the receiver be able to simulate the statistics of certain restricted measurements. In the case of quantum identification, these are $(\varphi, 1-\varphi)$ for arbitrary rank-one projectors $\varphi=|\varphi\rangle\langle\varphi| \in \mathcal{P}(K)$. They are the measurements which allow the receiver to ask the (quantum) question: "Is the state equal to $\varphi$ or orthogonal to it?" Obviously, in quantum theory this question cannot be answered with certainty, but for each test state it yields a characteristic distribution. The quantum-ID task above is about reproducing this distribution.

Note that we can always concatenate a blind or visible quantum ID-code for the Hilbert space $K$ with a fingerprinting set of pure states in $K$, to obtain a classical ID-code in the sense of Definition 1. This is because in fingerprinting the encodings are pure states $\psi_{i}$ and the tests precisely the POVMs $\left(\psi_{i}, 1-\psi_{i}\right)$. Hence, as the cardinality of the fingerprinting set is exponential in the dimension $|K|, M(n, \epsilon)$ and $M_{v}(n, \epsilon)$ can be at most exponential in $n$.

Definition 10. For a quantum channel $\mathcal{N}$, the blind, respectively visible, quantum ID-capacity is defined as

$$
\begin{aligned}
Q_{\mathrm{ID}}(\mathcal{N}) & :=\inf _{\epsilon>0} \liminf _{n \rightarrow \infty} \frac{1}{n} \log M(n, \epsilon), \\
Q_{\mathrm{ID}, \mathrm{v}}(\mathcal{N}) & :=\inf _{\epsilon>0} \liminf _{n \rightarrow \infty} \frac{1}{n} \log M_{v}(n, \epsilon) .
\end{aligned}
$$

If we leave out the qualifier, the quantum ID-capacity is by default the blind variety.

Note that by definition and the above remark,

$$
Q_{\mathrm{ID}}(\mathcal{N}) \leq Q_{\mathrm{ID}, \mathrm{v}}(\mathcal{N}) \leq C_{\mathrm{ID}}(\mathcal{N}) .
$$

The first quantum ID-capacity that had been determined was for the ideal qubit channel:

Theorem 11 (Winter [32]). For the noiseless channel $\mathrm{id}_{A}$ on Hilbert space $A$, there exists a (blind) quantum ID-code with error $\epsilon$ and encoding a space $K$ of dimension $|K| \geq C(\epsilon)|A|^{2}$, for some universal function $C(\epsilon)>0$.

As a consequence, $Q_{\mathrm{ID}}\left(\mathrm{id}_{2}\right)=Q_{\mathrm{ID}, \mathrm{v}}\left(\mathrm{id}_{2}\right)=2$, twice the quantum transmission capacity. 
In view of this theorem, we gain at least 2 in capacity for each noiseless qubit we use additionally to the given channel. This motivates the following definition.

Definition 12 (Hayden/Winter [22]). For a quantum channel $\mathcal{N}$, the amortized (blind/visible) quantum ID-capacity is defined as

$$
\begin{aligned}
Q_{\mathrm{ID}}^{\mathrm{am}}(\mathcal{N}) & :=\sup _{k} Q_{\mathrm{ID}}\left(\mathcal{N} \otimes \mathrm{id}_{k}\right)-2 \log k, \\
Q_{\mathrm{ID}, \mathrm{v}}^{\mathrm{am}}(\mathcal{N}) & :=\sup _{k} Q_{\mathrm{ID}, \mathrm{v}}\left(\mathcal{N} \otimes \mathrm{id}_{k}\right)-2 \log k,
\end{aligned}
$$

respectively.

The blind quantum ID-capacities are among the best understood, thanks to recently made conceptual progress, which we review in the next section. We will then also ask the question how much amortization is required. This is formalized in the usual way: Namely, for a rate $Q \leq Q_{\mathrm{ID}}^{\mathrm{am}}(\mathcal{N})$, we say that $A$ is an achievable amortization rate if there exist $k_{n}$ for all $n$, such that

$$
\liminf _{n \rightarrow \infty} \frac{1}{n}\left(Q_{\mathrm{ID}}\left(\mathcal{N}^{\otimes n} \otimes \operatorname{id}_{k_{n}}\right)-2 \log k_{n}\right) \geq Q \quad \text { and } \quad \limsup _{n \rightarrow \infty} \frac{1}{n} \log k_{n} \leq A,
$$

giving rise to an achievable quantum ID-rate/amortization region, viz. a tradeoff between $Q$ and $A$. Similarly of course for the visible variant.

\section{$3 \quad$ Weak Decoupling Duality}

The fundamental insight about quantum information transmission, which allowed an understanding of the quantum capacity as we have it today, is the decoupling principle: for a channel to permit (approximate) error correction it is necessary and sufficient that it leaks (almost) no information to the environment in the sense that the complementary channel $\widehat{\mathcal{N}}$ is close to constant. To be precise, $\operatorname{id}_{A^{\prime}} \otimes \widehat{\mathcal{N}}$ should map an entangled test state $\Phi^{A^{\prime} A}$ to $\approx \Phi^{A^{\prime}} \otimes \sigma^{E}$, where the approximation is with respect to the trace norm on density operators. In practice, to define capacities it is enough to demand this for the maximally entangled test state between the code space and a reference system 27 .

This condition is compactly expressed as saying that $\widehat{\mathcal{N}}$ is $\approx\left[\sigma^{E}\right]$ in the so-called diamond norm, the completely bounded version of the naive superoperator norm. Here, $\left[\sigma^{E}\right]$ denotes the constant channel mapping every input to $\sigma^{E}$. Because of this connection to completely bounded norms, we call channels with the above property completely forgetful or decoupling.

Indeed, it is well-known that this is a much stronger condition than $\widehat{\mathcal{N}}(\rho) \approx$ $\sigma^{E}$ for all input states $\rho$ on $A$. Cf. 20] for some instances of this effect relevant to quantum information processing. There, it is shown how to construct channels that are only (approximately) forgetful (or weakly decoupling), but far from completely forgetful.

To state the following conceptual points about blind(!) quantum ID-codes, it is useful to fix an encoding $\operatorname{cptp} \operatorname{map} \mathcal{E}: \mathcal{L}(K) \longrightarrow \mathcal{L}(A)$ and to combine it 
with the noisy channel, $\mathcal{N}^{\prime}=\mathcal{N} \circ \mathcal{E}$, for which we choose a Stinespring dilation $V: K \hookrightarrow B \otimes F$. The quantum ID-code is now the entire input space $K$ of this effective new channel, together with the previously given operators $D_{\varphi}$ on $B$. The next result states that just as quantum error correctability of $\mathcal{N}^{\prime}$ is equivalent to $\widehat{\mathcal{N}}^{\prime}$ being decoupling [27/24], quantum identification is essentially equivalent to weak decoupling from the environment:

Theorem 13 (Hayden/Winter [22]). If $K$ is a $\epsilon$-quantum ID-code for the channel $\mathcal{N}^{\prime}$ with Stinespring dilation $V: K \hookrightarrow B \otimes F$, then the complementary channel $\widehat{\mathcal{N}}^{\prime}$ is approximately forgetful:

$$
\forall|\varphi\rangle,|\psi\rangle \in K \quad \frac{1}{2}\left\|\widehat{\mathcal{N}}^{\prime}(\varphi)-\widehat{\mathcal{N}}^{\prime}(\psi)\right\|_{1} \leq \delta:=7 \sqrt[4]{\epsilon}
$$

Conversely, if $\widehat{\mathcal{N}^{\prime}}$ is approximately forgetful with error $\delta$, then the trace-norm geometry is approximately preserved by $\mathcal{N}^{\prime}$ :

$$
\forall|\varphi\rangle,|\psi\rangle \in S \quad 0 \leq\|\varphi-\psi\|_{1}-\left\|\mathcal{N}^{\prime}(\varphi)-\mathcal{N}^{\prime}(\psi)\right\|_{1} \leq \epsilon:=4 \sqrt{2 \delta} .
$$

If, in addition, the nonzero eigenvalues of the environment's states $\widehat{\mathcal{N}^{\prime}}(\varphi)$ lie in the interval $[\mu, \lambda]$ for all $|\varphi\rangle \in K$, then one can construct an $\eta$-quantum ID-code for $\mathcal{N}^{\prime}$ (i.e. a set of operators $D_{\varphi}$ for all $|\varphi\rangle \in K$ as in Definition 9), with $\eta:=7 \delta^{1 / 8} \sqrt{\lambda / \mu}$.

Remark 14. While it would be desirable to eliminate the eigenvalue condition at the end of the theorem, the condition is fairly natural in this context. If the environment's states $\widehat{\mathcal{N}^{\prime}}(\varphi)$ are very close to a single state $\sigma^{F}$ for all $|\varphi\rangle \in K$, then all the $V|\varphi\rangle$ are very close to being purifications of $\sigma^{F}$, meaning that they differ from one another only by a unitary plus a small perturbation. If $\sigma^{F}$ is the maximally mixed state or close to it, then the assumption will be satisfied. In the asymptotic iid setting we are looking at this turns to be the case.

This characterization of quantum ID-codes (albeit "only" blind ones) allows the determination of capacities by a random coding argument, for which only the weak decoupling has to be verified. The above duality theorem is not only the basis for the direct but also for the converse part(s) of the following capacity theorem.

Theorem 15 (Hayden/Winter [22]). For a quantum channel $\mathcal{N}$, its (blind) quantum ID-capacity is given by

$$
\begin{aligned}
Q_{\mathrm{ID}}(\mathcal{N}) & =\lim _{n \rightarrow \infty} \frac{1}{n} Q_{\mathrm{ID}}^{(1)}\left(\mathcal{N}^{\otimes n}\right), \text { where } \\
Q_{\mathrm{ID}}^{(1)}(\mathcal{N}) & \left.=\sup _{|\phi\rangle}\left\{I(A: B)_{\rho} \text { s.t. } I(A\rangle B\right)_{\rho}>0\right\},
\end{aligned}
$$

where $|\phi\rangle$ is the purification of an input state to $\mathcal{N}, \rho^{A B}=(\mathrm{id} \otimes \mathcal{N}) \phi$ and $I(A: B)_{\rho}=S\left(\rho^{A}\right)+S\left(\rho^{B}\right)-S\left(\rho^{A B}\right)$ is the mutual information, and $\left.I(A\rangle B\right)_{\rho}=$ 
$S\left(\rho^{B}\right)-S\left(\rho^{A B}\right)$ the coherent information (which already appeared in eq. (2)). We declare the sup to be 0 if the set above is empty. In particular, $Q_{\mathrm{ID}}(\mathcal{N})=0$ if and only if $Q(\mathcal{N})=0$.

Furthermore, the amortized quantum ID-capacity equals

$$
Q_{\mathrm{ID}}^{\mathrm{am}}(\mathcal{N})=\sup _{|\phi\rangle} I(A: B)_{\rho}=C_{E}(\mathcal{N}),
$$

the entanglement-assisted classical capacity of $\mathcal{N}$ [12].

Remark 16. Let us say that a channel $\mathcal{N}$ has "sufficiently low noise" if for an input state $|\phi\rangle$ maximizing $I(A: B)_{\rho}, \rho=(\operatorname{id} \otimes \mathcal{N}) \phi$, it holds that $\left.I(A\rangle B\right)_{\rho}>0$. This is motivated by the fact that in this case the channel has positive quantum capacity. Also, for any channel, $\mathcal{N} \otimes \mathrm{id}_{k}$ has sufficiently low noise if $k$ is chosen large enough; likewise $p \mathcal{N}+(1-p)$ id if $p>0$ is small enough.

In that case, the above tells us $Q_{\mathrm{ID}(\mathcal{N})}=Q_{\mathrm{ID}}^{\mathrm{am}}(\mathcal{N})=\sup _{|\phi\rangle} I(A: B)_{\rho}$, which is an additive, single-letter formula.

This theorem also shows that amortized and non-amortized quantum IDcapacities are different - indeed, any channel $\mathcal{N}$ with vanishing quantum capacity also has $Q_{\mathrm{ID}}(\mathcal{N})=0$, whereas $Q_{\mathrm{ID}}^{\mathrm{am}}(\mathcal{N})=0$ only for trivial channels. In particular this implies that $Q_{\text {ID }}$ is not additive. In [22] it is in fact proven that certain channels require a positive rate of amortization to attain or even to approximate $Q_{\mathrm{ID}}^{\mathrm{am}}$. The example analyzed there is the qubit erasure channel

$$
\begin{aligned}
\mathcal{E}_{q}: \mathcal{L}\left(\mathbb{C}^{2}\right) & \longrightarrow \mathcal{L}\left(\mathbb{C}^{3}\right) \\
\rho & \longmapsto(1-q) \rho \oplus q|*\rangle * \mid,
\end{aligned}
$$

which will serve us again in the following section. To be precise, for $0 \leq q<\frac{1}{2}$, the channel has sufficiently low noise and no amortization is required. For $\frac{1}{2} \leq q \leq 1$ instead, an amortized rate of at least $2 q-1$ qubits per channel use are necessary.

On the other hand, for all symmetric channels, i.e. those with $E=B$ in the Stinespring representation and $\mathcal{N}=\widetilde{\mathcal{N}}$, whereas quantum capacity and hence $Q_{\mathrm{ID}}$ are zero, only a vanishing rate of amortization is necessary to attain $Q_{\mathrm{ID}}^{\mathrm{am}}$. This is because they have $I(A\rangle B)_{\rho}=0$ for every input state, so arbitrarily little is required to make the coherent information positive.

This includes qc-channels with rank-one POVM $\left(M_{y}\right)_{y \in \mathcal{Y}}$, and the noiseless classical bit channel

$$
\overline{\mathrm{id}}_{2}: \rho \longmapsto \sum_{b=0,1}|b\rangle\langle b|\rho| b\rangle\langle b| .
$$

The latter implies that also cq-channels $\mathcal{N}$ only require a vanishing rate of amortization to attain $Q_{\mathrm{ID}}^{\operatorname{am}}(\mathcal{N})=C(\mathcal{N})=C^{(1)}(\mathcal{N})$ : This is because we can use $n \gg 1$ copies of $\mathcal{N}$, with appropriate encoding and decoding, to simulate $(C(\mathcal{N})-o(1)) n$ almost noiseless classical bits. This also shows that the rate $C(\mathcal{N})$ is attainable for all channels $\mathcal{N}$ as an amortized quantum ID-rate, with vanishing rate of amortization. 
In fact, inspection of the proof of the direct part of Theorem 15) (Thm. 12 in [22]) reveals that for the noiseless classical channel $\overline{\mathrm{id}}_{2}$, a constant amount of amortization is enough, hence the same for all cq-channels, and also for certain rank-one POVM qc-channels, namely those for which the outputs $\mathcal{N}(\tau)$ and $\tilde{\mathcal{N}}(\tau)$ for the maximally mixed input state $\tau_{A}$ are themselves maximally mixed. Because then the typicality arguments in the proof, which deal with eigenvalue fluctuations around the inverse exponential of the entropy, are unnecessary.

Remark 17. The previous observations show that the amortized quantum IDcapacity of a cq-channel $\mathcal{N}$ (which equals its classical capacity) can be achieved by visible, non-amortized codes:

$$
Q_{\mathrm{ID}, \mathrm{v}}(\mathcal{N})=Q_{\mathrm{ID}}^{\mathrm{am}}(\mathcal{N})=C(\mathcal{N})=C^{(1)}(\mathcal{N}) .
$$

Indeed, choose a sequence of amortized quantum ID-codes for $n$ uses of the channel, with amortized noiseless communication of a system of dimension $t=$ $o(\log n)$. Then, whatever the code produces as the input state $\omega=\mathcal{E}(\psi)$ to the channel $\mathcal{N}^{\otimes n} \otimes \mathrm{id}_{t}$, the effect is the same if we first dephase the input to $\mathcal{N}^{\otimes n}$ as the channel is cq, so w.l.o.g.

$$
\omega=\sum_{x^{n}} p_{x^{n}}\left|x^{n}\right\rangle\left\langle x^{n}\right| \otimes \omega_{x^{n}}
$$

with states $\omega_{x^{n}} \in \mathcal{S}\left(\mathbb{C}^{t}\right)$. The latter can be described classically to good approximation using $o(n)$ bits 21132, which can be communicated by $o(n)$ uses of the channel (if we exclude the trivial case of zero capacity).

This then is the visible scheme: the encoding of state $\psi$ is to sample from the distribution $p_{x^{n}}$ and sending $\left|x^{n}\right\rangle\left\langle x^{n}\right|$ through $\mathcal{N}^{\otimes n}$, and to send a classical description of $\omega x^{n}$ via $\mathcal{N}^{\otimes o(n)}$. The receiver creates then $\omega_{x^{n}}$ in addition to the other channel output, and otherwise uses the measurement $D_{\varphi}$ from the amortized ID-code.

That the capacity cannot be larger than $C(\mathcal{N})$ follows from eq. (3) and Theorem 4.

On the other hand, $Q_{\mathrm{ID}}(\mathcal{N})=0$ by Theorem [15, so we obtain a separation between blind and visible quantum ID-capacity, a question left open in [32.

We close this section by pointing out that $Q_{\mathrm{ID}}^{\mathrm{am}}$ is one of only two fully understood identification capacities so far: it has a single letter formula which can be evaluated efficiently and it is additive. The other one is the classical IDcapacity of a quantum channel with "coherent feedback" (meaning that in each use of the channel, the environment of the Stinespring isometry ends up with the sender), which we did not discuss here; the interested reader is referred to 33 .

\section{From $Q_{\text {ID }}$ to $C_{\text {ID }}$}

As pointed out in section 2 concatenating a quantum ID-code (blind or visible) with the fingerprinting construction (Example 6), yields a classical ID-code of 
asymptotically the same rate. Hence,

$$
\begin{gathered}
C_{\mathrm{ID}}(\mathcal{N}) \geq Q_{\mathrm{ID}, \mathrm{v}}(\mathcal{N}) \geq\left\{\begin{array}{l}
Q_{\mathrm{ID}}(\mathcal{N}), \\
C(\mathcal{N}),
\end{array}\right. \\
C_{\mathrm{ID}}^{\mathrm{am}}(\mathcal{N}) \geq Q_{\mathrm{ID}}^{\mathrm{am}}(\mathcal{N})=C_{E}(\mathcal{N}),
\end{gathered}
$$

where the amortized classical ID-capacity is defined analogously to the quantum variant.

Perhaps we do not find the amortized classical ID-capacity that interesting, but at least we get lots of channels for which $C_{\mathrm{ID}}(\mathcal{N}) \geq C_{E}(\mathcal{N})$, namely all sufficiently low noise channels and of course all cq-channels. This bound improves on the earlier best bound

$$
C_{\mathrm{ID}}(\mathcal{N}) \geq \max \{C+2 Q:(Q, C) \text { jointly achievable }\},
$$

the right hand side of which is always $\leq C_{E}(\mathcal{N})$. For example for the erasure channel $\mathcal{E}_{q}$, the quantum-classical-capacity region is known [16] to be

$$
\operatorname{conv}\left\{(0,0),(0,1-q),\left((1-2 q)_{+}, 0\right)\right\},
$$

so the above maximization yields

$$
C_{\mathrm{ID}}\left(\mathcal{E}_{q}\right) \geq \begin{cases}2-4 q & \text { for } 0 \leq q \leq \frac{1}{3} \\ 1-q & \text { for } \frac{1}{3} \leq q \leq 1\end{cases}
$$

Our new bound instead is

$$
C_{\mathrm{ID}}\left(\mathcal{E}_{q}\right) \geq \begin{cases}2-2 q & \text { for } 0 \leq q<\frac{1}{2} \\ 1-q & \text { for } \frac{1}{2} \leq q \leq 1\end{cases}
$$

which is strictly better in the interval $\left[0, \frac{1}{2}\right)$.

\section{Conclusion and Open Questions}

As it should have become clear from the above exposition, identification theory in the quantum setting is an enormously fruitful area, much more so even than the classical version, if only because we have at least five natural capacities. And we did not yet even touch upon a general theory of information transfer in quantum information, or rather how quantum information would fit into this far-reaching vision 223, these aspects still awaiting development.

At the same time the subject of identification via quantum channels is wide open, with most of the questions implied in the original papers [25]10/32] remaining unsolved, despite significant progress over the last decade. In particular, it turned out that the quantum identification task lent itself much more easily to the currently available techniques, and that the recent progress satisfyingly shed a fresh light on the older, and seemingly more elementary classical identification task. The following seven broad open problems are recommended to the reader's attention. 
1. Surely the biggest open problem is to determine the classical ID-capacity $C_{\mathrm{ID}}(\mathcal{N})$ of a general quantum channel, and to study its properties, such as additivity etc. Even obtaining non-trivial upper bounds would be a worthy goal. Note that practically all transmission capacities of a channel are upper bounded by its entanglement-assisted capacity, by way of the Quantum Reverse Shannon Theorem 12[13]14 through simulation of the channel by noiseless communication and unlimited shared entanglement. This argument is not available here since entanglement or even common randomness have an impact on the ID-capacities.

In fact, the few cases for which $C_{\mathrm{ID}}$ is known are consistent with the idea that it is always equal to the entanglement-assisted classical capacity of the channel [32. One might speculate that $C_{\mathrm{ID}}(\mathcal{N}) \geq Q_{\mathrm{ID}, \mathrm{v}}(\mathcal{N}) \geq C_{E}(\mathcal{N})$ be true for all channels, seeing that for sufficiently low noise we can prove it, and that it is true for the amortized classical ID-capacity. The erasure channel $\mathcal{E}_{q}$ discussed in section 4 is already an excellent test case for this idea.

2. Is there a deeper, operational, reason why the amortized quantum ID-capacity equals the entanglement-assisted classical(!) capacity of a channel? In the derivation of 22 this comes out naturally as a result of the analysis, but almost as an accident, and it seems difficult to connect it to [12]...

3 . Is the simultaneous ID-capacity $C_{\mathrm{ID}}^{\operatorname{sim}}(\mathcal{N})$ equal to the non-simultaneous version $C_{\mathrm{ID}}(\mathcal{N})$ ? I suspect that they are different, possibly even for the noiseless qubit channel (see Theorem 7 and subsequent remarks). In such a case we face another problem to determine $C_{\mathrm{ID}}^{\mathrm{sim}}(\mathcal{N})$. When studying simultaneous ID-codes, Löber's technical condition in Theorem 4 deserves special attention, as it precludes using the entire input state space of the iid channels. A very interesting case to study will be (rank-one POVM) qc-channels as there any identification code is per se simultaneous. For these channels we know the amortized quantum ID-capacity (it is the entanglement-assisted classical capacity, which evaluates to $\log |A|$ ), and that amortization rate 0 is sufficient to achieve it, in some cases even a constant amount. In fact, it would be interesting to know whether the visible quantum ID-capacity for these channels is the same - cf. the case of cq-channels discussed in Remark 17 -; this would evidently prove $C_{\mathrm{ID}}(\mathcal{N}) \geq \log |A|$ for all these channels $\mathcal{N}$, whereas it is known that the classical capacity $C(\mathcal{N})$ for many of them is much smaller.

4. The role of amortization is extremely interesting: For the quantum IDcapacity it makes for a quasi-superactivation effect, since a vanishing rate of it (i.e. an arbitrary small rate of noiseless communication) can turn a capacity 0 channel into one of positive capacity. It is possible that vanishing rate of amortization likewise has an impact on classical ID-capacities - see the example of qc-channels discussed in the previous point.

Finally, in 22] only the non-triviality of amortization (and only for the erasure channels) was proved. How to characterize the quantum ID-rate vs. amortization rate tradeoff?

5. We have seen that the visible quantum ID-capacity can be larger than the blind variant, indeed the former can be positive while the latter is 0 for cq- 
channels. Let us note that the distinction visible/blind can also be made in the quantum transmission game, and there it is far from clear whether there will be a difference in capacities, see 32 .

6. We did not comment much on the role of shared correlations in the identification game, indeed referring the reader to 3233, where also the impact of feedback is discussed. However, in Proposition 8 we saw that not only is the classical ID-capacity of common randomness (in the presence of negligible communication) equal to 1 per bit, but it increases the rate of any given ID-code by 1 per bit. We also know that the classical ID-capacity of shared entanglement is 2 per ebit, but it is open whether we can augment a given ID-code with entanglement to increase its rate by 2 per ebit.

7. Finally: All the known upper bounds on classical ID-capacities are in fact strong converses. Does the strong converse also hold for (visible, blind, amortized, etc) quantum ID-capacities? This question seems to require new techniques to be answered.

\section{Acknowledgements}

I have thought about identification in quantum information theory for quite some time, going back all the way to the days of my $\mathrm{PhD}$, from which period date my hugely enjoyable mathematical interactions with Peter Löber, and of course with Rudolf Ahlswede, a sometimes terrifying but always, and ever newly, inspiring Doktorvater. In an article such as the present one it may be permitted to say that I miss him. Why, he keeps influencing my work even now!

Fortunately, later on others began to share my enthusiasm for quantum identification, most importantly Patrick Hayden. Much of the present review was only motivated by our recent collaboration.

I am or have been supported by a U.K. EPSRC Advanced Research Fellowship, the European Commission (STREP "QCS" and Integrated Project "QESSENCE"), the ERC Advanced Grant "IRQUAT", a Royal Society Wolfson Merit Award and a Philip Leverhulme Prize.

\section{References}

1. A. Abeyesinghe, P. Hayden, G. Smith and A. Winter. Optimal superdense coding of entangled states. IEEE Trans. Inf. Theory 52(8):3635-3641, 2006. arXiv quant-ph/0407061

2. R. Ahlswede. General theory of information transfer. Elec. Notes Discr. Math. 21:181-184, 2005 .

3. R. Ahlswede. General theory of information transfer: Updated. Discr. Applied Math. 156(9):1348-1388, 2008.

4. R. Ahlswede. On Concepts of Performance Parameters for Channels. In: R. Ahlswede et al. (eds.), General Theory of Information Transfer and Combinatorics, Lecture Notes in Computer Science, vol. 4123, pp. 639-663, Springer Verlag, Berlin Heidelberg 2006. 
5. R. Ahlswede and V. B. Balakirsky. Identification under random processes. Probl. Inf. Transm. 32(1):123-138, 1996.

6. R. Ahlswede, L. Bäumer, N. Cai, H. Aydinian, V. Blinovsky, C. Deppe and H. Mashurian. Introduction to the book. In: R. Ahlswede et al. (eds.), General Theory of Information Transfer and Combinatorics, Lecture Notes in Computer Science, vol. 4123, pp. 1-44, Springer Verlag, Berlin Heidelberg 2006.

7. R. Ahlswede and V. M. Blinovsky. Large Deviations in Quantum Information Theory. Probl. Inf. Transm. 39(4):373-379, 2003. (Translated form Problemy Peredachi Informatsii 39(4):63-70, 2003.)

8. R. Ahlswede and G. Dueck. Identification Via Channels. IEEE Trans. Inf. Theory 35(1):15-29, 1989.

9. R. Ahlswede and G. Dueck. Identification in the Presence of Feedback - A Discovery of New Capacity Formulas. IEEE Trans. Inf. Theory 35(1):30-36, 1989.

10. R. Ahlswede and A. Winter. Strong converse for identification via quantum channels. IEEE Trans. Inf. Theory 48(3):569-579, 2002. arXiv quant-ph/0012127

11. G. Aubrun, S. Szarek and E. Werner. Hastingss Additivity Counterexample via Dvoretzky's Theorem. Commun. Math. Phys. 305(1):85-97, 2011.

12. C. H. Bennett, P. W. Shor, J. A. Smolin and A. V. Thapliyal. EntanglementAssisted Capacity of a Quantum Channel and the Reverse Shannon Theorem. IEEE Trans. Inf. Theory 48(10):2637-2655, 2002.

13. C. H. Bennett, I. Devetak, A. W. Harrow, P. W. Shor and A. Winter. The quantum reverse Shannon theorem and resource tradeoffs for simulating quantum channels. arXiv[quant-ph]:0912.5537v2, 2012.

14. M. Berta, M. Christandl and R. Renner. The Quantum Reverse Shannon Theorem Based on One-Shot Information Theory. Commun. Math. Phys. 306(3):579-615, 2011. arXiv[quant-ph]:0912.3805.

15. H. Buhrman, R. Cleve, J. Watrous and R. de Wolf. Quantum fingerprinting. Phys. Rev. Lett. 87:167902, 2001. arXiv:quant-ph/0102001

16. I. Devetak and P. W. Shor. The Capacity of a Quantum Channel for Simultaneous Transmission of Classical and Quantum Information. Commun. Math. Phys. 256(2):287-303, 2005.

17. F. Dupuis, J. Florjanczyk, P. Hayden and D. Leung. Locking classical information arXiv[quant-ph]:1011.1612, 2010.

18. T.-S. Han and S. Verdú. New Results in the Theory of Identification via Channels. IEEE Trans. Inf. Theory 38(1):14-25, 1992.

19. T.-S. Han and S. Verdú. Approximation Theory of Output Statistics. IEEE Trans. Inf. Theory 39(3):752-772, 1993.

20. P. Hayden, D. Leung, P. W. Shor and A. Winter. Randomizing Quantum States: Constructions and Applications. Commun. Math. Phys. 250:371-391, 2004. arXiv:quant-ph/0307104

21. P. Hayden, D. Leung and A. Winter. Aspects of generic entanglement. Commun. Math. Phys. 265:95-117, 2006. arXiv:quant-ph/0407049.

22. P. Hayden and A. Winter. Weak Decoupling Duality and Quantum Identification. IEEE Trans. Inf. Theory 58(7):4914-4929, 2012. arXiv[quant-ph]:1003.4994.

23. A. W. Harrow, A. Montanaro and A. J. Short. Limitations on Quantum Dimensionality Reduction. In: L. Aceto, M. Henzinger and J. Sgall (eds.), Proc. ICALP 2011, Lecture Notes in Computer Science, vol. 6755, pp. 86-97, Springer Verlag, Berlin Heidelberg 2011.

24. D. Kretschmann, D. Schlingemann and R. F. Werner. The Information-Disturbance Tradeoff and the Continuity of Stinesprings Representation. IEEE Trans. Inf. Theory 54(4):1708-1717, 2008. 
25. P. Löber. Quantum channels and simultaneous ID coding. PhD thesis, Universität Bielefeld, Bielefeld (Germany), 1999. http://katalog.ub.uni-bielefeld.de/ title/1789755.

26. J. M. Renes. Approximate Quantum Error Correction via Complementary Observables. arXiv[quant-ph]:1003.1150, 2010.

27. B. Schumacher and M. D. Westmoreland. Approximate Quantum Error Correction. Quantum Inf. Proc. 1(1/2):5-12, 2002. arXiv quant-ph/0112106.

28. C. E. Shannon. A mathematical theory of communication. Bell Syst. Tech. J. 27:379-423 \& 623-656, 1948.

29. W. F. Stinespring. Positive Functions on $C^{*}$-Algebras. Proc. Amer. Math. Soc. $6(2): 211-216,1955$.

30. J. A. Tropp. User-Friendly Tail Bounds for Sums of Random Matrices. Found. Comput. Math. 12(4):389-434, 2012. arXiv[math.PR]:1004.4389, 2010.

31. M. M. Wilde. From Classical to Quantum Shannon Theory. arXiv[quantph]:1106.1445, 2011.

32. A. Winter. Quantum and classical message identification via quantum channels. In O. Hirota, editor, Festschrift "A. S. Holevo 60", pp. 171-188, Rinton Press, 2004. Reprinted in Quantum Inf. Comput. 4(6\&7):563-578, 2004. arXiv quant-ph/0401060

33. A. Winter. Identification via quantum channels in the presence of prior correlation and feedback. In: R. Ahlswede et al. (eds.), General Theory of Information Transfer and Combinatorics, Lecture Notes in Computer Science, vol. 4123, pp. 486-504, Springer Verlag, Berlin Heidelberg 2006. arXiv quant-ph/0403203 